\title{
Cancer incidence in the East Azerbaijan province of Iran in 2015-2016: results of a population-based cancer registry
}

\author{
Mohammad Hossein Somi ${ }^{1}$, Roya Dolatkhah ${ }^{2 *}$ (D, Sepideh Sepahi ${ }^{3}$, Mina Belalzadeh ${ }^{3}$, Jabraeil Sharbafi ${ }^{3}$, \\ Leila Abdollahi ${ }^{3}$, Azin Nahvijou ${ }^{4}$, Saeed Nemati ${ }^{4}$, Reza Malekzadeh ${ }^{5}$ and Kazem Zendehdel ${ }^{4,6^{*}}$
}

\begin{abstract}
Background: Few countries in the Middle East have a population-based cancer registry, despite a clear need for accurate cancer statistics in this region. We therefore established a registry in the East Azerbaijan province, the sixth largest province in northwestern Iran.

Methods: We actively collected data from 20 counties, 62 cities, and 44 districts for the period between $20^{\text {th }}$ March 2015 and $19^{\text {th }}$ March 2016 (one Iranian solar year). The CanReg 5 software was then used to estimate age-standardized incidence rates (ASRs) per 100,000 for all cancers and different cancer types.

Results: Data for 11,536 patients were identified, but we only analyzed data for 6655 cases after removing duplicates and non-residents. The ASR for all cancers, except non-melanoma skin cancer, was 167.1 per 100,000 males and 125.7 per 100,000 females. The most common cancers in men were stomach (ASR 29.7), colorectal (ASR 18.2), bladder (ASR 17.6), prostate (ASR 17.3), and lung (ASR 15.4) cancers; in women, they were breast (ASR 31.1), colorectal (ASR 13.7), stomach (13.3), thyroid (ASR 7.8), and esophageal (ASR 7.1) cancers. Both the death certificate rate (19.5\%) and the microscopic verification rate (65\%) indicated that the data for the cancer registry were of reasonable quality.

Conclusion: The results of the East Azerbaijan Population-based Cancer Registry show a high incidence of cancer in this province, especially gastrointestinal cancers.
\end{abstract}

Keywords: Cancer, Registry, Population-based, Iran, Incidence

\section{Background}

Cancer is a major cause of mortality and morbidity worldwide. According to the GLOBOCAN project, there were an estimated 14.1 million new cases and 8.2 million deaths from cancer globally in 2012 [1]. In 2013, the cancer incidence increased to 14.9 million new cancer cases [2]. Programs for population-based cancer registration are considered the gold standard method for collecting data on all new cancer cases. Moreover, these programs are essential for estimating the burden of cancer in specific populations, particularly when seeking to provide a framework for clarifying community-based risk

\footnotetext{
*Correspondence: dolatkhahr@tbzmed.ac.ir; kzendeh@gmail.com

${ }^{2}$ Hematology and Oncology Research Center, Tabriz University of Medical

Sciences, Tabriz, Iran

${ }^{4}$ Cancer Research Center, Cancer Institute of Iran, Tehran University of

Medical Sciences, Tehran, Iran

Full list of author information is available at the end of the article
}

factors and monitoring efforts to control cancer [2-4]. A National Pathology-based Cancer Registry Program was started in Iran in 2001 and was subsequently rolled out to all provinces, including the East Azerbaijan province [5]. However, there were large differences in the coverage of cancer registry program in all state and provinces of Iran, which made this program cover about $60-70 \%$ of all cancer cases data. [5-9] Etemadi et al. has described the numerous attempts to establish a population-based cancer registry in Iran $[9,10]$. One registry was maintained in the Golestan province [11-13], and its most recent results were published in the International Agency for Research on Cancer (IARC) monograph, "Cancer in Five Continents," in 2013 [14].

The first population-based studies in Iran estimated the incidence rates of all cancer types during 1996-2000 in the Golestan, Mazandaran, and Kerman provinces [15],

(c) The Author(s). 2018 Open Access This article is distributed under the terms of the Creative Commons Attribution 4.0 International License (http://creativecommons.org/licenses/by/4.0/), which permits unrestricted use, distribution, and reproduction in any medium, provided you give appropriate credit to the original author(s) and the source, provide a link to the Creative Commons license, and indicate if changes were made. The Creative Commons Public Domain Dedication waiver (http://creativecommons.org/publicdomain/zero/1.0/) applies to the data made available in this article, unless otherwise stated. 
for the Ardabil Province for 1996-1999 [7], and for the East Azerbaijan province for 2006-2007 [16]. The Iranian national population-based cancer registration is following a five-year plan, covering 14 provinces in its first phase, and is funded by the involved universities. The East Azerbaijan Population-based Cancer Registry (EA-PBCR) was established in 2006-2007, based on the goals and responsibilities directed by the chancellor of Tabriz University of Medical Sciences. In the EA-PBCR, high rates were reported for gastric cancer (ASR 37.6 per 100,000) and esophageal cancer (ASR 24.1 per 100,000) in men, and high rates were reported for breast cancer (ASR 23.5 per 100,000) in women [16]. Stomach cancer was the most common cancer in the north and northwest of Iran [17], as well as in the Ardabil Province for both men and women, and this region has since been considered a high-risk region for stomach cancer [18]. Indeed, according to Marzban et al., death from stomach cancer is up to six-fold higher in this region compared with southern Iran [7, 19].

In this study, we aimed to establish a standard population-based cancer registry in the East Azerbaijan province. The component aims were to create the necessary infrastructures, to initiate networking and collaborations between data sources, and to estimate the incidence rates for different cancers in this province. Research grants were obtained for stable funding, and we ensured proper governance and agreement among stakeholders. In this paper, we report the first results of the EA-PBCR.

\section{Methods}

\section{Data source}

Well-trained health information technology staff (the EA-PBCR team) collected data from 20 counties, 62 cities, and 44 districts about newly diagnosed cancers between March 20, 2015, and March 19, 2016 (one Iranian solar year). Sources included the following: pathology reports from 33 centers (including pathology and cytology reports); the medical records from 20 educational and private hospital, radiotherapy, and hematology centers; and 35 imaging centers. In several pathology laboratories and hospitals, data were obtained electronically from their information technology systems. Data for cancer-related deaths were obtained from death certificate registry data stored at Tabriz University of Medical Sciences. Also, we linked the last three years data of the national pathology-based cancer registry of Iran (INPCR), which led to the removal of additional duplicate records.

Data were imported to CanReg5 software (Persian Version), which is an open-source tool that allowed information input, storage, checking, and processing. The data were then divided into patient, tumor, and source tables. The following information was mandatory for inclusion in the study: first and last name, birth date, fathers name, national identification (NID) number, sex, place of residence, and date of diagnosis. The morphology (i.e., histology, behavior, and grade) and topography (primary site of origin) of the tumor, based on the International Classification of Diseases for Oncology Third Edition (ICD-O-3), were also reported [20]. Contact information was available for about $75 \%$ of cases, which will allow direct access to individual data in future studies. We then completed data entry, quality control, consistency checks, and basic analysis, using the Persian Version of CanReg5.

\section{Population of East Azerbaijan Province}

The East Azerbaijan province is one of 31 provinces of Iran and is the biggest and most populated province of northwest Iran (Fig. 1). It has the sixth largest population overall, and the largest Azeri ethnic population, in Iran. It covers an area of 45,620 $\mathrm{km}^{2}$ and had a total population of $3,911,278$ according to the 2015 national census in Iran. The EA-PBCR is held in the capital city, Tabriz.

Figure 2 shows a population pyramid for the East Azerbaijan province by age and sex group, emphasizing that the population was young, with a predominance of people aged 20-24 years. Population density can be seen to reduce gradually with increasing age, such that very few people aged 60-64 years and older were included. Table 1 shows the age-stratification groups in East Azerbaijan during the study period (Table 1, Fig. 2).

\section{Quality control}

The CanReg5 software was used to perform automatic checks for internal validity. We also performed quality control by manual and computerized validity checks of the cancer registry system based on the IARC criteria [21] in the cancer registry office of East Azerbaijan province. This involved assessing factors influencing comparability, validity, timeliness, and completeness [22-25]. Case duplication across the registry databases was checked in three steps to exclude repeated cases, as follows: 1) patient first name, family name; 2) patient first name, family name, and fathers' name; and 3) patient NID number. For multiple primary tumors we referred to IARC multiple primary rules for patients multiple primary cancers [26].

Also we discuss with an expert oncologist and pathologist, to determine primary tumors from invasion, metastasis, or recurrence cancer cases according the morphology and behavior of cancers, to avoid any missed and/or duplicated cancer data.

Microscopic verification (MV) is an important quality indicator for any cancer registry. We therefore automatically collected MV data from all available pathology reports (including histological and cytological reports) in laboratory databases. In some cases, these data were collected manually. Cases obtained from the death certificate registry without pathology or clinical data were reported as 


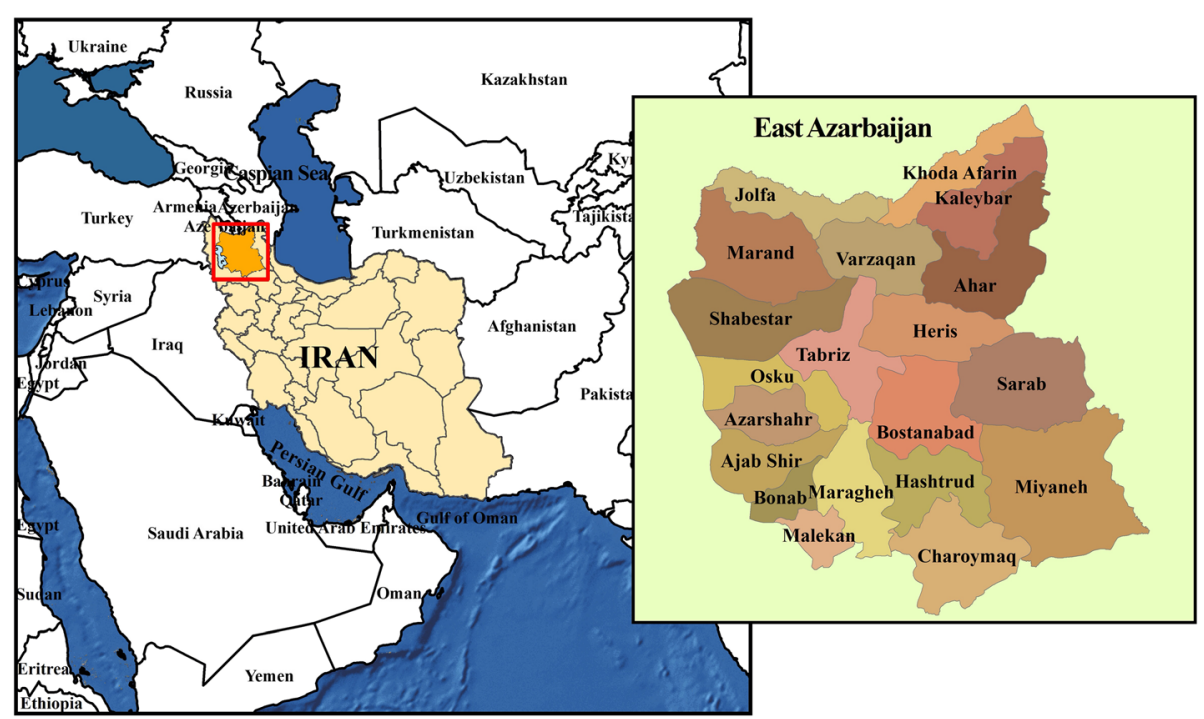

Fig. 1 Map of Iran showing East Azerbaijan (cities are shown in the inset)

death certificate only (DCO) cases. We then aimed to decrease the percentage of DCO cases (i.e., the DCO \%). First, we performed data linkage with several sources to identify histologic or clinical information. Second, we contacted patients or their relatives to increase the frequency of cases with MV or clinical data. Next, the incidence and mortality rates in the EA-PBCR for the period of study were used to calculate the mortality-to-incidence (M/I) ratio. The mortality data for this analysis were collected from our cancer registry, observed deaths (reports from follow-up records), or the national death certificate registry (reported deaths).

\section{Statistical analyses}

Descriptive data are presented as means \pm standard deviation or as numbers and percentages, unless otherwise stated. We estimated the frequencies, crude incidence rates, and age-standardized incidence rates (ASR) per 100,000 populations for all cancers, as well as for the different cancers among men and women. These ASRs per 100,000 were reported for each cancer in 18 strata of 5 -year age groups. The standard world population for 2000 was used to estimate the ASR [27].

\section{Results}

\section{Study sample}

Figure 3 shows the process and role of different data sources in the EA-PBCR. We merged 3954 records from pathology reports with 4416 reports from the medical records of hospital departments and 3166 reports from death certificates $(n=11536$ cases). After removing 3044 duplicates and 629 records for patients who were referred from neighboring provinces, 7863 records remained. Then, we removed an additional 1616 duplicate records

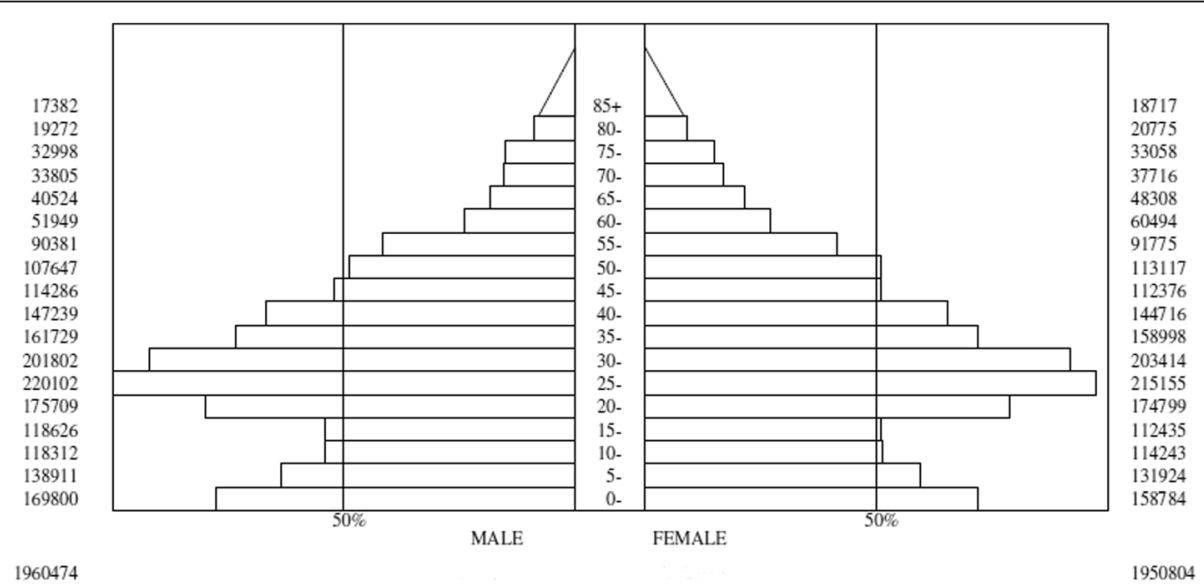

Fig. 2 The population distribution pyramid in the East Azerbaijan province, based on the national census report 2015-2016 
Table 1 The Exact Stratification Age Groups of East Azerbaijan, in 2015-2016

\begin{tabular}{|c|c|c|c|c|}
\hline \multicolumn{2}{|c|}{ Age Groups } & \multicolumn{3}{|c|}{ East Azerbaijan Population in 2015-2016 } \\
\hline & Male & Female & & \\
\hline & Number & Percent & Number & Percent \\
\hline $0-4$ & 169800 & 8.6 & 158784 & 8.13 \\
\hline 05-9 & 138911 & 7.08 & 131924 & 6.76 \\
\hline $10-14$ & 118312 & 6.03 & 114243 & 5.85 \\
\hline $15-19$ & 118626 & 6.05 & 112435 & 5.76 \\
\hline 20-24 & 175709 & 8.96 & 174799 & 8.96 \\
\hline $25-29$ & 220102 & 11.22 & 215155 & 11.02 \\
\hline $30-34$ & 201802 & 10.29 & 203414 & 10.42 \\
\hline $35-39$ & 161729 & 8.24 & 158998 & 8.15 \\
\hline $40-44$ & 147239 & 7.51 & 144716 & 7.41 \\
\hline $45-49$ & 114286 & 5.82 & 112376 & 5.76 \\
\hline $50-54$ & 107647 & 5.49 & 113117 & 5.79 \\
\hline $55-59$ & 90381 & 4.61 & 91775 & 4.70 \\
\hline $60-64$ & 51949 & 2.64 & 60494 & 3.10 \\
\hline $65-69$ & 40524 & 2.06 & 48308 & 2.47 \\
\hline 70-74 & 33805 & 1.72 & 37716 & 1.93 \\
\hline 75-79 & 32998 & 1.68 & 33058 & 1.69 \\
\hline 80-84 & 19272 & 0.98 & 20775 & 1.06 \\
\hline $85<$ & 17382 & 0.88 & 18717 & 0.95 \\
\hline Total & 1960474 & 100 & 1950804 & 100 \\
\hline
\end{tabular}

after linkage with INPCR (6247 remained). An additional 408 cases were included during follow-up investigations of patients who were referred to other cities. Finally, the cohort comprised 6655 incident cases that met the inclusion criteria (Fig. 3).

\section{Data quality Validity and completeness}

After rigorous assessment, $65.1 \%$ of the cases were found to have MV, including histology (63.2\%) and cytology (1.9\%) results. Furthermore, $15.2 \%$ of reports were collected based on clinical data, including medical records $(14 \%)$ and imaging reports $(1.2 \%)$. The remaining data were collected from the cause of death registry (19.5\%) and from autopsy records $(0.2 \%)$, producing a final DCO\% of $19.7 \%$.

\section{The $M / I$ ratio}

The M/I ratio was about 59\% (3954/6655), indicating that we received a sufficient number of records from the cause of death registry and that the $\mathrm{DCO} \%$ was reasonable.

\section{Death certificate notification}

The total number of cancer deaths in the cause of death registry for East Azerbaijan was 3166. After removing duplicates by linking the database with current and previous cancer registries, we searched for tumor information from alternative sources to improve the validity of our results because the DCO\% was high (19.7\%). Following this, 1267 cases remained for analysis (1267/ $6655=19.0 \%)$. Data from death certificates and contact

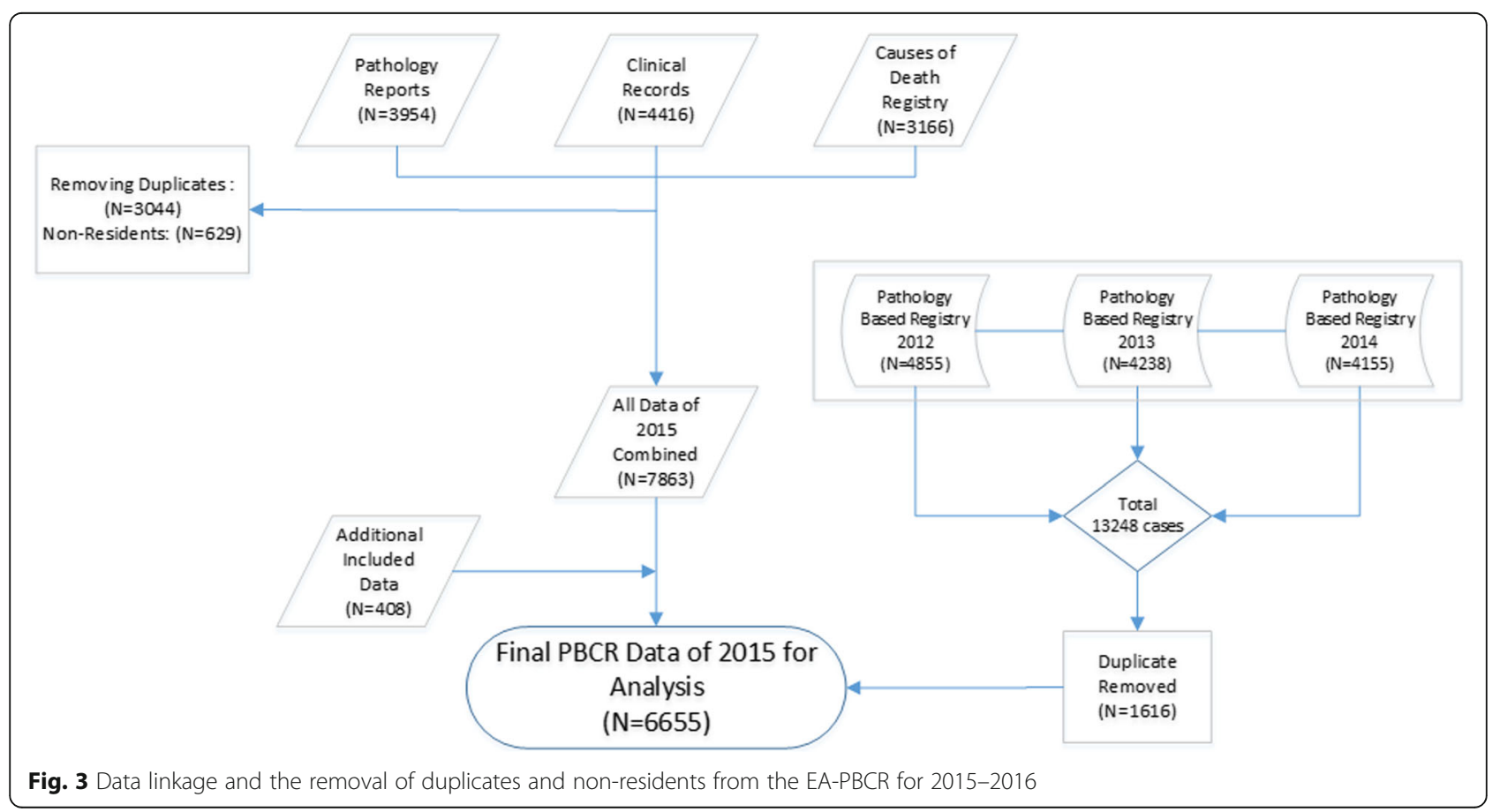


with hospitals and patients' relatives uncovered the clinical or pathology reports for a further 177 patients. These additions allowed us to change the basis of the associated diagnoses $([1267-177] / 6655=1090 / 6655=$ 16.4\%). Thus, although the initial data had a higher $\mathrm{DCO} \%$, this decreased after linkage with the different databases, after contacting relatives to remove cases diagnosed previously, and after clarifying the clinical and histological information.

\section{Cancer incidence}

Of the 6655 new cancer cases in during the Iranian solar year under study, males accounted for 3728 (56.02 \%) and females accounted for 2927 (43.98\%), giving a male-to-female ratio of 1.27 . The overall mean age was $60.97 \pm 17.21$ years, but this was higher for men (63.68 \pm 16.68 years) and lower for women (57.53 \pm 17.27 years).

Except for non-melanoma skin cancer (ICD-O-3 code C44), the crude incidence rates per 100,000 were 175.2 for men and 141.0 for women. The ASRs per 100,000 for all cancers were 167.1 for men and 125.7 for women. The five most common cancers were stomach (ASR 29.7), colorectal (ASR 18.2), bladder (ASR 17.6), prostate (ASR 17.3), and lung (ASR 15.4) for men; and for women, these were breast (ASR 31.1), colorectal (ASR 13.7), stomach (ASR 13.3), thyroid (ASR 7.8), and esophageal (ASR 7.1). Table 2 and Figs. 4, 5 summarize these results, while Table 3 shows the morphologic distributions and frequencies of the most common cancers in the province during 2015-2016 (Tables 2, 3, Figs. 4, 5).

\section{Discussion}

The EA-PBCR was established to allow accurate estimates of annual statistics in the province by collecting information on incident cases of cancer from various sources. The main data sources in this study were pathology reports, medical records, and death certificate. However, records from additional sources were also used to improve the completeness and validity of the results, including from radiotherapy and chemotherapy departments, imaging facilities, and hematology laboratories. The ASRs for all cancers in 2015, excluding non-melanoma skin cancer, were 167.1 per 100,000 for males and 125.7 per 100,000 for females. Our data show that the five most common cancers in this region, by sex, were stomach, colorectal, bladder, prostate, and lung in men, and were breast, colorectal, stomach, thyroid, and esophageal in women. Importantly, both the $\mathrm{DCO} \%$ and the MV rate indicate that the EA-PBCR data were of reasonable quality. Our results are also comparable with those of GLOBOCAN 2012 and of the latest report of Golestan Province population-based cancer registry (Table 4), which are the most recent and reliable to have been published for Iran $[1,28]$.

The EA-PBCR has followed international standards for data collection and reporting since its inception. For example, we linked available data from different sources to improve the validity and completeness of our results. Indeed, the $\mathrm{MV}$ rate, the $\mathrm{DCO} \%$, and the $\mathrm{M} / \mathrm{I}$ ratio were comparable to those reported for middle-income countries [29-32]. Another advantage was that we created a unique NID number as a mandatory item for use by hospitals, cause of death registries, and most laboratories. This number allows for deterministic data linkage and for improvements in data validity and completeness. However, the EA-PBCR is still in its infancy, and we faced some important limitations. The $\mathrm{DCO} \%$, for example, was still high and was difficult to decrease in the first year. Also NID was not available for all databases, and we will try to improve this in our next reports.

Table 2 ASRs for the top ten major cancers in males and females in East Azerbaijan Province in 2015-2016

\begin{tabular}{|c|c|c|c|c|c|c|c|c|c|}
\hline \multicolumn{5}{|l|}{ Male } & \multicolumn{5}{|l|}{ Female } \\
\hline Site (ICD-O-3) & No. of Cases & Proportion (\%) & $C I R^{b}$ & $\overline{A S R^{c}}$ & Site (ICD-O-3) & No. of Cases & Proportion (\%) & CIR & $\overline{A S R}$ \\
\hline Stomach (C16) & 610 & 17.8 & 31.1 & 29.7 & Breast (C50) & 681 & 24.8 & 34.9 & 31.1 \\
\hline Colorectal (C18-21) & 366 & 10.7 & 18.7 & 18.2 & Colorectal (C18-21) & 299 & 10.8 & 15.4 & 13.7 \\
\hline Bladder (C67) & 356 & 10.4 & 18.2 & 17.6 & Stomach (C16) & 302 & 11.0 & 15.5 & 13.3 \\
\hline Prostate (C61) & 369 & 10.7 & 18.8 & 17.3 & Thyroid (C73) & 175 & 6.4 & 9.0 & 7.8 \\
\hline Lung (C33-34) & 320 & 9.3 & 16.3 & 15.4 & Esophagus (C15) & 157 & 5.7 & 8.0 & 7.1 \\
\hline Leukemia (C91-95) & 196 & 5.7 & 10 & 9.6 & Leukemia (C91-95) & 117 & 4.2 & 6 & 5.5 \\
\hline Esophagus (C15) & 172 & 5.0 & 8.8 & 8.0 & Lung (C33-34) & 117 & 4.3 & 6.0 & 5.0 \\
\hline $\begin{array}{l}\text { Lymphoma } \\
(\mathrm{C} 81-85,88,90,96)\end{array}$ & 126 & 3.7 & 6.4 & 6.4 & Ovary (C56) & 97 & 3.5 & 5.0 & 4.8 \\
\hline Liver (C22) & 115 & 3.3 & 5.9 & 5.9 & Bladder (C67) & 87 & 3.2 & 4.5 & 3.9 \\
\hline Brain\& CNS (C70-72) & 111 & 3.2 & 5.7 & 5.3 & Liver (C22) & 82 & 3.0 & 4.2 & 3.5 \\
\hline
\end{tabular}

International Classification of Diseases for Oncology Third Edition code

${ }^{b}$ Crude Incidence Rate

'Age-standardized Incidence Rate 

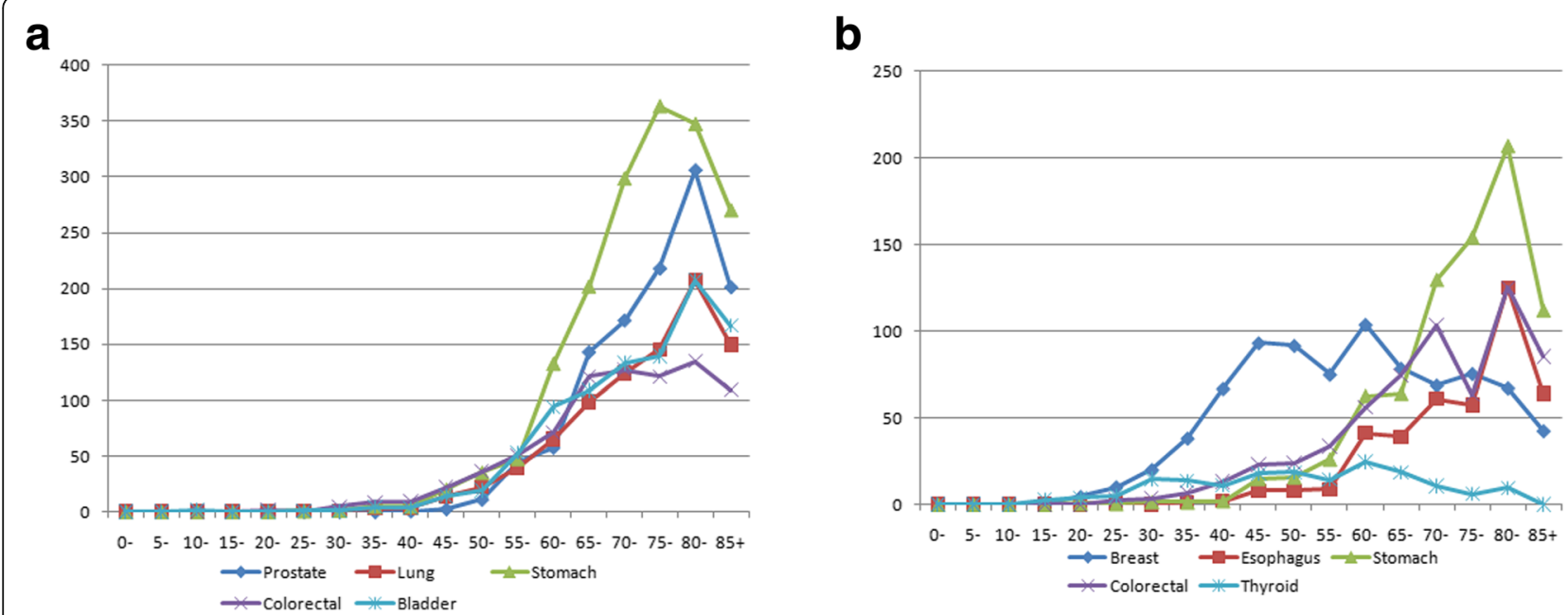

Fig. 4 a ASRs for the top five major cancers in males. $\mathbf{b}$ ASRs for the top five major cancers in females

We believe that the quality indicators will improve rapidly as we move forward. To improve the quality of the data produced from these sources, we have called for greater collaboration with the cause of death registry and with hospital managers.
According to this study, gastric cancer-which is the fourth most common cancer worldwide-was the most common cancer among males (ASR 29.7 per 100,000) and the third most common cancer among females (ASR 13.3 per 100,000). A few studies of the incidence

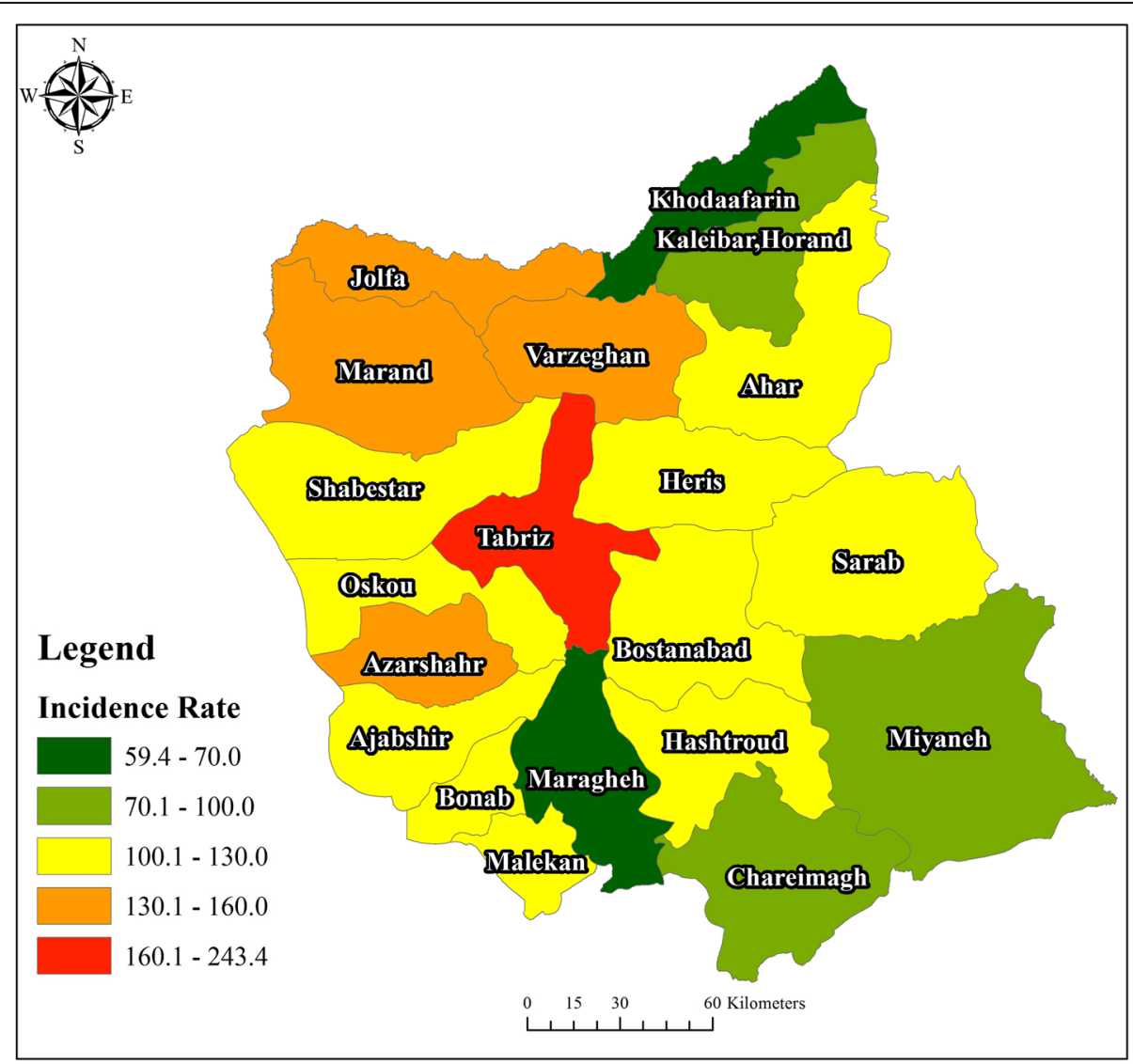

Fig. 5 Crude incidence rates of all cancers in East Azerbaijan cities 
Table 3 Morphologic Distribution and Frequency of most common cancers in East Azerbaijan Province in 2015-2016

\begin{tabular}{|c|c|c|c|c|c|c|c|c|c|c|c|}
\hline \multicolumn{2}{|l|}{ Cancer Site } & \multirow[t]{2}{*}{ Frequent } & \multirow[t]{2}{*}{ Percent } & \multicolumn{2}{|l|}{ Cancer Site } & \multirow[t]{2}{*}{ Frequent } & \multirow[t]{2}{*}{ Percent } & \multicolumn{2}{|l|}{ Cancer Site } & \multirow[t]{2}{*}{ Frequent } & \multirow[t]{2}{*}{ Percent } \\
\hline $\begin{array}{l}\text { Cardiac Gastric } \\
\text { Cancer }\end{array}$ & Gender & & & $\begin{array}{l}\text { Esophageal } \\
\text { Cancer }\end{array}$ & Gender & & & Breast Cancer & Gender & & \\
\hline \multirow{2}{*}{$\begin{array}{l}\text { Neoplasm, } \\
\text { Malignant }\end{array}$} & Male & 4 & 7.0 & \multirow{2}{*}{$\begin{array}{l}\text { Neoplasm, } \\
\text { Malignant }\end{array}$} & Male & 14 & 8.1 & \multirow{2}{*}{$\begin{array}{l}\text { Neoplasm, } \\
\text { Malignant }\end{array}$} & Male & 1 & 0.14 \\
\hline & Female & 2 & 8.0 & & Female & 9 & 5.7 & & Female & 43 & 6.09 \\
\hline \multirow{2}{*}{$\begin{array}{l}\text { Epithelial Tumor, } \\
\text { Malignant }\end{array}$} & Male & 1 & 1.8 & \multirow{2}{*}{$\begin{array}{l}\text { Epithelial Tumor, } \\
\text { Malignant }\end{array}$} & Male & 4 & 2.3 & \multirow[t]{2}{*}{ Carcinoma } & Male & - & - \\
\hline & Female & - & - & & Female & 2 & 1.3 & & Female & 28 & 3.96 \\
\hline \multirow{2}{*}{$\begin{array}{l}\text { Squamous Cell } \\
\text { Carcinoma }\end{array}$} & Male & 1 & 1.8 & \multirow{2}{*}{$\begin{array}{l}\text { Squamous Cell } \\
\text { carcinoma }\end{array}$} & Male & 131 & 75.7 & \multirow{2}{*}{$\begin{array}{l}\text { Papillary } \\
\text { Carcinoma }\end{array}$} & Male & 1 & 0.14 \\
\hline & Female & 8 & 32.0 & & Female & 141 & 88.7 & & Female & - & - \\
\hline \multirow{2}{*}{$\begin{array}{l}\text { Adenocarcinoma/ } \\
\text { NOS }\end{array}$} & Male & 24 & 42.1 & \multirow{2}{*}{$\begin{array}{l}\text { Adenocarcinoma/ } \\
\text { NOS }\end{array}$} & Male & 9 & 5.2 & \multirow{2}{*}{$\begin{array}{l}\text { Squamous Cell } \\
\text { Carcinoma }\end{array}$} & Male & - & - \\
\hline & Female & 8 & 32.0 & & Female & 5 & 3.1 & & Female & 1 & 0.14 \\
\hline Adenocarcinoma, & Male & 19 & 33.3 & Adenocarcinoma, & Male & 12 & 6.9 & Adenocarcinoma & Male & - & - \\
\hline & Female & 4 & 16.0 & & Female & 1 & 6 & & Female & 1 & 0.14 \\
\hline Adenocarcinoma, & Male & 4 & 7.0 & Adenocarcinoma, & Male & - & - & Tubular & Male & - & - \\
\hline & Female & 1 & 4.0 & & Female & - & - & & Female & 1 & 0.14 \\
\hline Mucinous & Male & 1 & 1.8 & Mucinous & Male & 1 & 6 & Mucinous & Male & - & - \\
\hline Adenocarcinoma & Female & - & - & Adenocarcinoma & Female & - & - & Adenocarcinoma & Female & 1 & 0.14 \\
\hline Sebaceous & Male & - & - & Sebaceous & Male & - & - & Signet Ring Cell & Male & - & - \\
\hline Adenocarcinoma & Female & 1 & 4.0 & arcinoma & Female & - & - & C & Female & 1 & 0.14 \\
\hline Signet Ring Cell & Male & 3 & 5.3 & Signet Ring Cell & Male & 1 & 6 & Ductal Carcinoma & Male & 12 & 1.6 \\
\hline Carcınoma & Female & 1 & 4.0 & Car & Female & - & - & & Female & 587 & 83.14 \\
\hline Others & Male & - & - & Others & Male & 1 & 6 & Medullary & Male & - & - \\
\hline & Female & - & - & & Female & 1 & 6 & & Female & 8 & 1.13 \\
\hline Total & Male & 57 & 100.0 & Total & Male & 173 & 100.0 & Lobular Carcinoma & Male & - & - \\
\hline & Female & 25 & 100.0 & & Female & 159 & 100.0 & & Female & 17 & 2.40 \\
\hline $\begin{array}{l}\text { Non-Cardiac } \\
\text { Gastric Cancer }\end{array}$ & & & & Colorectal Cancer & & & & $\begin{array}{l}\text { Infiltrating Ductal } \\
\text { and Lobular }\end{array}$ & Male & - & - \\
\hline Neoplasm, & Male & 55 & 9.9 & Neoplasm, & Male & 20 & 5.4 & & Female & 1 & 0.14 \\
\hline & Female & 31 & 11.1 & & Female & 33 & 10.7 & Phylloides Tumor & Male & - & - \\
\hline Epithelial Tumor, & Male & 7 & 1.3 & Epithelial Tumor, & Male & 1 & .3 & & Female & 2 & 0.28 \\
\hline & Female & 6 & 2.1 & & Female & - & - & Myeloid Sarcoma & Male & - & - \\
\hline Squamous Cell & Male & 3 & .5 & Squamous Cell & Male & 2 & .5 & & Female & 1 & 0.14 \\
\hline & Female & 1 & .4 & & Female & 2 & .7 & Unknown & Male & - & - \\
\hline Adenocarcinoma/ & Male & 281 & 50.4 & Adenocarcinoma/ & Male & 330 & 89.9 & & Female & - & - \\
\hline & Female & 147 & 52.5 & & Female & 254 & 82.7 & Total & Male & 14 & 100.0 \\
\hline Adenocarcinoma, & Male & 155 & 27.8 & Adenocarcinoma, & Male & - & - & & Female & 692 & 100.0 \\
\hline & Female & 71 & 25.4 & & Female & 1 & .3 & Lung Cancer & & & \\
\hline Adenocarcinoma, & Male & 18 & 3.2 & Adenocarcinoma, & Male & - & - & asm, & Male & 88 & 19.86 \\
\hline & Female & 6 & 2.1 & & Female & 1 & .3 & & Female & 40 & 9.02 \\
\hline Mucinous & Male & 4 & .7 & Mucinous & Male & 6 & 1.6 & Malignant Tumor, & Male & 2 & 0.45 \\
\hline A & Female & 1 & .4 & A & Female & 3 & 1.0 & & Female & - & - \\
\hline Sebaceous & Male & - & - & Sebaceous & Male & - & - & Malignant Tumor, & Male & - & - \\
\hline & Female & - & - & & Female & - & - & & Female & 1 & 0.22 \\
\hline Signet Ring Cell & Male & 26 & 4.7 & Signet Ring Cell & Male & 4 & 1.1 & Carcinoma & Male & 11 & 2.48 \\
\hline & Female & 13 & 4.6 & & Female & 4 & 1.3 & & Female & 5 & 1.12 \\
\hline Others & Male & 2 & .4 & Others & Male & 4 & 1.2 & Small Cell & Male & 12 & 2.70 \\
\hline
\end{tabular}


Table 3 Morphologic Distribution and Frequency of most common cancers in East Azerbaijan Province in 2015-2016 (Continued)

\begin{tabular}{|c|c|c|c|c|c|c|c|c|c|c|c|}
\hline \multirow{2}{*}{$\begin{array}{l}\text { Cancer Site } \\
\text { Cardiac Gastric } \\
\text { Cancer }\end{array}$} & \multirow{2}{*}{\multicolumn{2}{|c|}{$\begin{array}{l}\text { Frequent } \\
\text { Gender }\end{array}$}} & \multirow[t]{2}{*}{ Percent } & \multirow{2}{*}{$\begin{array}{l}\text { Cancer Site } \\
\text { Esophageal } \\
\text { Cancer }\end{array}$} & \multirow[b]{2}{*}{ Gender } & \multirow[t]{2}{*}{ Frequent } & \multirow[t]{2}{*}{ Percent } & \multicolumn{2}{|l|}{ Cancer Site } & \multirow[t]{2}{*}{ Frequent } & \multirow[t]{2}{*}{ Percent } \\
\hline & & & & & & & & Breast Cancer & Gender & & \\
\hline & Female & 3 & 1.1 & & Female & 6 & 1.8 & Carcinoma, NOS & Female & 3 & 0.67 \\
\hline \multirow[t]{2}{*}{ Unknown } & Male & 6 & 1.1 & \multirow[t]{2}{*}{ Unknown } & Male & - & - & \multirow{2}{*}{$\begin{array}{l}\text { Non-small Cell } \\
\text { Carcinoma }\end{array}$} & Male & 5 & 1.12 \\
\hline & Female & 1 & .4 & & Female & - & - & & Female & 1 & 0.22 \\
\hline \multirow[t]{2}{*}{ Total } & Male & 551 & 100.0 & \multirow[t]{2}{*}{ Total } & Male & 367 & 100.0 & \multirow{2}{*}{$\begin{array}{l}\text { Squamous Cell } \\
\text { Carcinoma }\end{array}$} & Male & 172 & 38.81 \\
\hline & Female & 279 & 100.0 & & Female & 307 & 100.0 & & Female & 54 & 12.18 \\
\hline Thyroid Cancer & & & & Prostate & & & & \multirow[t]{2}{*}{ Adenocarcinoma } & Male & 27 & 6.09 \\
\hline \multirow{2}{*}{$\begin{array}{l}\text { Neoplasm, } \\
\text { Malignant }\end{array}$} & Male & 4 & 1.70 & \multirow{2}{*}{$\begin{array}{l}\text { Neoplasm, } \\
\text { Malignant }\end{array}$} & Male & 19 & 5.09 & & Female & 9 & 2.03 \\
\hline & Female & 8 & 3.40 & & Female & - & - & \multirow{2}{*}{$\begin{array}{l}\text { Carcinoid Tumor, } \\
\text { NOS }\end{array}$} & Male & - & - \\
\hline \multirow{2}{*}{$\begin{array}{l}\text { Papillary } \\
\text { Carcinoma }\end{array}$} & Male & 9 & 3.82 & \multirow[t]{2}{*}{ Carcinoma } & Male & 78 & 20.91 & & Female & 4 & 0.90 \\
\hline & Female & 28 & 11.91 & & Female & - & - & Bronchiolo-Alveolar & Male & 3 & 0.67 \\
\hline \multirow[t]{2}{*}{$\begin{array}{l}\text { Squamous Cell } \\
\text { Carcinoma }\end{array}$} & Male & - & - & \multirow{2}{*}{$\begin{array}{l}\text { Carcinoma, } \\
\text { Undifferentiated } \\
\text {,NOS }\end{array}$} & Male & 1 & 0.26 & $\begin{array}{l}\text { Adenocarcinoma, } \\
\text { NOS }\end{array}$ & Female & - & - \\
\hline & Female & 1 & 0.42 & & Female & - & - & Signet Ring Cell & Male & 1 & 0.22 \\
\hline Papillary & Male & 41 & 17.44 & Squamous Cell & Male & 1 & 0.26 & Car & Female & - & - \\
\hline enocarcınoma & Female & 101 & 42.97 & & Female & - & - & Sarcoma, NOS & Male & - & - \\
\hline Oxyphilic & Male & 1 & 0.42 & Transitional Cell & Male & 3 & 0.80 & & Female & 1 & 0.22 \\
\hline Adenocarcinoma & Female & 6 & 2.55 & Carcinoma, NOS & Female & - & - & Spindle cell & Male & 1 & 0.22 \\
\hline Follicular & Male & 2 & 0.85 & Papillary & Male & 3 & 0.80 & & Female & - & - \\
\hline Adenocarcinoma & Female & 13 & 5.52 & $\begin{array}{l}\text { Iransitional Lell } \\
\text { Carcinoma }\end{array}$ & Female & - & - & Total & Male & 322 & 100.0 \\
\hline Insular Carcinoma & Male & - & - & Adenocarcinoma & Male & 266 & 71.31 & & Female & 118 & 100.0 \\
\hline & Female & 1 & 0.42 & & Female & - & - & Bladder Cancer & Gender & & \\
\hline Papillary Carcinoma, & Male & 3 & 1.27 & Adenocarcinoma, & Male & 2 & 0.53 & Neoplasm, & Male & 17 & 3.82 \\
\hline & Female & 13 & 5.53 & & Female & - & - & Malıgnant & Female & 2 & 0.44 \\
\hline Medullary Carcinoma & Male & 1 & 0.42 & Unknown & Male & - & - & Transitional Cell & Male & 217 & 48.76 \\
\hline $\begin{array}{l}\text { with Amyloid } \\
\text { Stroma }\end{array}$ & Female & - & & & Female & - & - & & Female & 49 & 11.01 \\
\hline Mixed Medullary & Male & 2 & .84 & Total & Male & 373 & 100.0 & Papillary & Male & 1 & 0.22 \\
\hline & Female & - & & & Female & - & - & & Female & 1 & 0.22 \\
\hline Medullary & Male & - & & & & & & Squamous Cell & Male & 1 & 0.22 \\
\hline Carcinoma, NOS & Female & 3 & 1.27 & & & & & Carcinoma & Female & - & - \\
\hline Total & Male & 62 & 100.0 & & & & & Papillary & Male & 117 & 26.29 \\
\hline & Female & 173 & 100.0 & & & & & $\begin{array}{l}\text { Transitional } \\
\text { Cell Carcinoma }\end{array}$ & Female & 34 & 7.64 \\
\hline & & & & & & & & Adenocarcinoma & Male & 2 & 0.44 \\
\hline & & & & & & & & & Female & 1 & 0.22 \\
\hline & & & & & & & & Carcinoid tumor, & Male & 3 & 0.66 \\
\hline & & & & & & & & & Female & - & - \\
\hline & & & & & & & & Total & Male & 358 & 100.0 \\
\hline & & & & & & & & & Female & 87 & 100.0 \\
\hline
\end{tabular}

of gastrointestinal cancer have been performed in the East Azerbaijan province [5, 16, 33-35]. In the most recent survey of gastrointestinal cancer in this province between 2007 and 2011, the ASR for gastric cancer was raised in both men (26 per 100,000) and women (11.6 per
$100,000)$, and it was the second-leading cause of death (10.4\% of all deaths) [34]. Other research showed that stomach cancer has been the most common cancer in north and northwestern Iran over the past 30 years [17, 18, 36]. The highest ASRs for stomach cancer in men 
Table 4 Comparing of age standardized incidence rate (ASR) for most common cancers in Iran (GLOBOCAN 2012), Golestan Province Population Based Cancer Registry Results (2004-2013), and East Azerbaijan Population Based Cancer Registry Results (2015-2016)

\begin{tabular}{|c|c|c|c|c|c|c|c|c|c|c|}
\hline \multirow[t]{2}{*}{ Cancer Site } & \multirow[t]{2}{*}{ Gender } & \multicolumn{3}{|c|}{ GLOBOCAN 2012 Iran } & \multicolumn{3}{|c|}{$\begin{array}{l}\text { Golestan Province Population } \\
\text { Based Cancer Registry } \\
\text { Results (2004-2013) } \\
\end{array}$} & \multicolumn{3}{|c|}{$\begin{array}{l}\text { East Azerbaijan Population } \\
\text { Based Cancer Registry } \\
\text { Results (2015-2016) }\end{array}$} \\
\hline & & Number & \% Total & ASR & Number & \% Total & ASR & Number & \% Total & ASR \\
\hline \multirow[t]{2}{*}{ Esophagus } & Male & 2898 & 6.5 & 9.0 & 1221 & 12.3 & 21.6 & 171 & 5.0 & 8.9 \\
\hline & Female & 2445 & 6.1 & 8.0 & 930 & 10.5 & 16.8 & 157 & 5.7 & 8.3 \\
\hline \multirow[t]{2}{*}{ Stomach } & Male & 6640 & 14.8 & 20.6 & 1576 & 15.8 & 27.4 & 610 & 17.7 & 32.9 \\
\hline & Female & 3020 & 7.6 & 9.7 & 686 & 7.8 & 12.0 & 301 & 10.9 & 15.6 \\
\hline \multirow[t]{2}{*}{ Colorectal } & Male & 3811 & 8.5 & 11.6 & 911 & 9.2 & 14.9 & 412 & 12.0 & 22.7 \\
\hline & Female & 3352 & 8.4 & 10.5 & 715 & 8.1 & 11.6 & 332 & 12.1 & 17.8 \\
\hline \multirow[t]{2}{*}{ Leukemia } & Male & 2338 & 5.2 & 6.9 & 817 & 8.2 & 12.3 & 196 & 5.7 & 9.6 \\
\hline & Female & 1588 & 4.0 & 4.7 & 599 & 6.7 & 9.1 & 117 & 4.2 & 5.5 \\
\hline \multirow[t]{2}{*}{ Lymphoma } & Male & 2611 & 5.9 & 7.3 & 567 & 5.7 & 8.0 & 126 & 3.7 & 6.4 \\
\hline & Female & 1703 & 4.2 & 4.9 & 333 & 3.8 & 4.5 & 69 & 2.4 & 3.6 \\
\hline \multirow[t]{2}{*}{ Lung } & Male & 3307 & 7.4 & 10.3 & 907 & 9.1 & 16.0 & 322 & 9.4 & 17.4 \\
\hline & Female & 1581 & 4.0 & 5.0 & 362 & 4.1 & 6.2 & 118 & 4.3 & 6.1 \\
\hline \multirow[t]{2}{*}{ Melanoma (Skin) } & Male & 295 & 0.7 & 0.9 & 661 & 6.6 & 11.4 & 9 & 0.3 & 0.4 \\
\hline & Female & 239 & 0.6 & 0.7 & 416 & 4.7 & 7.1 & 3 & 0.1 & 0.1 \\
\hline \multirow[t]{2}{*}{ Breast } & Male & - & - & - & - & - & - & 14 & 0.4 & 0.7 \\
\hline & Female & 9795 & 24.5 & 28.1 & 2121 & 24.0 & 29.3 & 683 & 24.7 & 36.0 \\
\hline \multirow[t]{2}{*}{ Ovary } & Male & - & - & - & - & - & - & - & - & - \\
\hline & Female & 1637 & 4.1 & 4.8 & 400 & 4.5 & 5.7 & 97 & 3.5 & 5.4 \\
\hline \multirow[t]{2}{*}{ Prostate } & Male & 4111 & 9.2 & 12.6 & 690 & 6.9 & 12.0 & 369 & 10.7 & 19.4 \\
\hline & Female & - & - & - & & & & - & - & - \\
\hline \multirow[t]{2}{*}{ Bladder } & Male & 4277 & 9.5 & 13.2 & 529 & 5.3 & 9.2 & 356 & 10.4 & 19.8 \\
\hline & Female & 1066 & 2.7 & 3.4 & & & & 87 & 3.2 & 4.6 \\
\hline \multirow[t]{2}{*}{ Brain, CNS } & Male & 1699 & 3.8 & 4.6 & 475 & 4.8 & 6.9 & 111 & 3.2 & 5.9 \\
\hline & Female & 1358 & 3.4 & 3.9 & 369 & 4.2 & 5.3 & 69 & 2.5 & 3.7 \\
\hline \multirow[t]{2}{*}{ Thyroid } & Male & 513 & 1.1 & 1.4 & - & - & - & 63 & 1.8 & 3.1 \\
\hline & Female & 1512 & 3.8 & 4.0 & - & - & - & 173 & 6.3 & 8.6 \\
\hline \multirow[t]{2}{*}{ All cancers excl. non-melanoma skin cancer } & Male & 44838 & 100.0 & 134.7 & 10577 & 100.0 & 175.0 & 3439 & 100.0 & 186.0 \\
\hline & Female & 39991 & 100.0 & 120.1 & 9230 & 100.0 & 142.4 & 2761 & 100.0 & 146.1 \\
\hline
\end{tabular}

(51.8; 95\% CI 47.8-55.8) and women (24.9; 95\% CI 21.527.2) per 100,000 were reported for the Ardebil province in 2010 [7, 18, 37]. Gastric cancer is also known to be the most common cause of cancer-related death in the country, although there is a reported six-fold geographic variation in mortality rates between northwestern and southern Iran [19]. The elevated risk of stomach cancer incidence and mortality in the northwestern region has been linked to the higher prevalence of Helicobacter pylori infection [38, 39], tobacco and opium use, and dietary factors [40-45]. A well-designed case-control study in northern Iran showed a positive association between red meat consumption and the risk of gastric cancer [46].
The incidence rates of bladder cancer (ASR 17.6 per 100,000 ) and lung cancer (ASR 15.4 per 100,000), which are associated with tobacco smoking and opium use [47, 48], were also high in the male population. Studies in the 1980s in Southern Iran [49] and recent studies from Golestan in northwestern Iran [50] have shown that opium is a more important risk factor for bladder cancer than tobacco. Opium use is also common in East Azerbaijan (unpublished data from a Persian cohort) and may play an important role in the etiology of bladder cancer in this province.

The ASR of lung cancer was higher, based on clinical diagnosis or DCO, in the EA-PBCR than the rate 
reported for Iran in GLOBOCAN 2012 (i.e., 15.4 versus 10.3 per 100,000 ) [1]. In the Golestan population-based cancer registry, the incidence of lung cancer was also higher than the Iranian average for males $(A S R=17.5$ per 100,000) [14]. Therefore, it appears that both the pathology-based registry [6, 9], and GLOBOCAN 2012 may have underestimated the true incidence of lung cancer in Iran. The ASR of lung cancer was also low among females in both Tabriz (ASR 5 per 100,000) and Golestan (ASR 5.6 per 100,000) [14]. A recent analysis of the "National Surveys of Risk Factor of Non-Communicable Diseases (STEPS)" [sic] showed that the prevalence of cigarette smoking was high in northwestern Iran, and that $23.7 \%$ of men in the East Azerbaijan province reported being smokers [51]. By comparison, the prevalence of cigarette smoking is reported to be much lower (12.4\%) among men in the Bushehr province of southern Iran, where water pipe smoking is preferred; here, the prevalence of water pipe use was reportedly $10 \%$ for men and $14.8 \%$ for women [51]. Therefore, the high incidence rates for lung and bladder cancers in the East Azerbaijan province may be linked to cigarette smoking, highlighting the importance of tobacco control measures in this region. Opium use is also an emerging risk factor for both respiratory tract and lung cancers [52], with recent cohort studies from Golestan revealing the important role of opium in such cancers [53].

The incidence rates of colorectal, prostate, and breast cancer are increasing in low- and middle-income countries [54, 55], including Iran [33, 56-58]. This study showed that the incidence rates for colorectal cancer were high for both men $(20.2$ per 100,000$)$ and women (16.2 per 100,000). Likewise, high incidence rates were identified for breast cancer in women (ASR 31 per $100,000)$ and for prostate cancer in men (ASR 17.3 per 100,000) in the East Azerbaijan province. The World Health Organization Office in the Eastern Mediterranean Region recently published a series of recommendations for early cancer detection [59], and although they did not recommend the need for organized colorectal or breast cancer screening, they did emphasize the need for early diagnosis through improvements in public awareness and management of symptomatic patients. This recommendation might be valid for now, but we need to be prepared for a more comprehensive colonoscopy and mammography screening program in the future, when the incidence rates for these cancers can be expected to increase, making screening programs the most cost-effective options. Worldwide, prostate cancer screening is not recommended because of the high false-positive rate when using the prostate-specific antigen [59].

Based on the national cancer registry results in Iran, thyroid and laryngeal cancers are among the most common cancers of the head and neck, with oral and thyroid cancers being predominant in females [60]. Our results also showed a higher incidence of thyroid cancer in females (ASR = 7.78); indeed, it was the fourth most common cancer after breast, colorectal, and stomach cancers, with an ASR that was increased compared with a previous report from East Azerbaijan [16]. Significant associations between increased thyroid cancer incidence and lifestyle risk factors, radiation exposure, smoking, and obesity have been reported in a few studies [61-63]. The difference in the pattern of thyroid cancer incidence in our female cohort demands more comprehensive studies, which we have already started. The results of these will be presented soon.

Esophageal cancer may be one of the least common cancers in most countries, but it is the fourth most common cancer in Iran, being the second-leading cause of cancer and cancer-related mortality. The highest incidence rates have been reported for northern provinces [13]. According to the most recent results of studies in this region, there was a significant decrease in the incidence of esophageal cancer [34, 64]. However, we still observed a high incidence of esophageal cancer, especially in females, which was the fifth most common cancer among women. Compared with previous reports from the cancer registry, the ASR for esophageal cancer has shown a decrease in East Azerbaijan over the last decade $[16,34]$.

Finally, we found that the incidence of gynecological cancers, including ovarian (ASR 4.8 per 100,000), cervical (ASR 1.58 per 100,000), and endometrial (ASR 2.8 per 100,000) cancers, were low in East Azerbaijan. The extremely low incidence of cervical cancer was comparable to that previously reported for the Ardabil Province [7]. The prevalence of human papillomavirus (HPV) infection in women has been reported at $6.1 \%$ in the East Azerbaijan province [65], compared with $7 \%$ in Iran in general [66]. This comparatively low prevalence may be attributable to the local Muslim culture, in which more people engage in safer sexual behaviors and fewer people have multiple sexual partners [16, 66-68]. Given the low incidence of cervical cancer, neither a cervical screening program nor a HPV vaccination program would be cost-effective in this area. However, sexual behavior is changing in younger generations, and this may lead to an increase in the rate of HPV infection and the risk of cervical cancer in the future. Therefore, regular monitoring of the incidence of HPV infection and cervical cancer is warranted [69].

\section{Conclusions}

In this study, we presented the most current and reliable data for cancer incidence in northwestern Iran. The quality of the EA-PBCR is promising, and we believe that maintaining and developing this registry will 
establish a high-quality population-based cancer registry in the region. The results from the EA-PBCR could also be used to estimate cancer-specific incidence and mortality rates in Iran and neighboring countries, making it a potentially invaluable resource for the planning and monitoring of cancer control programs and for the delivery of reliable epidemiological research. Moving forward, our main aims will be to perform survival analyses of the most common cancers in East Azerbaijan, to ensure that the registry continues to exist, and to further develop the follow-up system.

\section{Abbreviations \\ ASR: Age-standardized incidence rate; Cl: Confidence interval; CLIN: Clinical; DCO: Death certificate only; EA-PBCR: East Azerbaijan Population-based Can- cer Registry; HPV: Human papillomavirus; IARC: International Agency for Research on Cancer; ICD-O-3: International Classification of Diseases for Oncology, Third Edition; MV: Microscopic verification; NID: National identification number; STEPS: National Surveys of Risk Factor of Non- Communicable Diseases}

\section{Acknowledgments}

This study was supported by Tabriz University of Medical Sciences. We would like to acknowledge the technical support of the Cancer Institute at Tehran University of Medical Sciences, Tehran, Iran. Finally, Dr Robert Sykes (www.doctored.org.uk) provided technical editing services during several drafts of this manuscript.

\section{Funding}

This research project was supported and funded by Tabriz University of Medical Sciences (Grant Number: IR.TBZMED.REC.1395.1333).

\section{Availability of data and materials}

The datasets used and/or analyzed during the study will be made available by the corresponding author following a reasonable request.

\section{Disclosure}

The abstract of this work has been presented as "Oral Presentation" in: 1. International Association of cancer Registry Annual Conference, 17-19 October 2017, Utrecht, Netherlands, by the title of: "Result of the New Initiative to established East Azerbaijan Population Based cancer Registry (EA-PBCR) in 2015"

2. International Congress on Cancer Prevention \& Early Detection Integration of Research \& Action , 28-30 January 2017, Tehran, Iran, by the title of : "Preliminary Results of East Azerbaijan Population-Based Cancer registry during 2015-2016"Second Comprehensive Report of Cancer Incidence (Archives of Iranian Medicine, Volume 20, Number 1, January 2017 (Suppl. 1) S18)

\section{Authors' contributions}

$\mathrm{RD}$ and $\mathrm{KZ}$ designed the study, supervised the project, data abstraction, and data analysis; they prepared the initial draft and finalized it based on comments from the other authors. MHS, RM, and AN contributed to the study design and provided technical support and consultation during the conduct of the registry. SS and SN participated in the data collection, data linkage, and cleaning and analysis of the registry data. MB, JS, and LA (registrars) conducted data abstraction and cleaning. All authors reviewed each manuscript draft, participated in interpreting the results, and approved the final version.

\section{Ethics approval and consent to participate}

The ethics committee of Tabriz University of Medical Sciences approved this project (Code: IR.TBZMED.REC.1395.1333). All patient data and records were stored confidentially. Consent to participate was not applicable.

\section{Consent for publication}

Not applicable.

\section{Competing interests}

The authors declare that they have no competing interests.

\section{Publisher's Note}

Springer Nature remains neutral with regard to jurisdictional claims in published maps and institutional affiliations.

\section{Author details}

${ }^{1}$ Liver and Gastrointestinal Diseases Research Center, Tabriz University of Medical Sciences, Tabriz, Iran. ${ }^{2}$ Hematology and Oncology Research Center, Tabriz University of Medical Sciences, Tabriz, Iran. ${ }^{3}$ Cancer Registry Office, Tabriz University of Medical Sciences, Tabriz, Iran. ${ }^{4}$ Cancer Research Center, Cancer Institute of Iran, Tehran University of Medical Sciences, Tehran, Iran. ${ }^{5}$ Digestive Oncology Research Center, Digestive Disease Research Institute, Tehran University of Medical Sciences, Tehran, Iran. ${ }^{6}$ Cancer Biology Research Center, Cancer Institute of Iran, Tehran University of Medical Sciences, Tehran, Iran.

Received: 11 January 2018 Accepted: 12 October 2018

Published online: 19 November 2018

\section{References}

1. Ferlay J, Soerjomataram I, Dikshit R, et al. Cancer incidence and mortality worldwide: sources, methods and major patterns in GLOBOCAN. Int J Cancer. 2015;136(5):E359-86.

2. Global Burden of Disease Cancer C, Fitzmaurice C, Dicker D, et al. The Global Burden of Cancer. JAMA Oncol. 2015;1(4):505-27.

3. Parkin DM. The evolution of the population-based cancer registry. Nat Rev Cancer. 2006;6(8):603-12.

4. Roshandel G, Boreiri M, Sadjadi A, Malekzadeh R. A diversity of cancer incidence and mortality in West Asian populations. Ann Glob Health. 2014; 80(5):346-57.

5. Hossein Somi M, Mirinezhad K, Farhang S, et al. Gastrointestinal cancer occurrence in East Azarbaijan: a five year study from North Western Iran. Asian Pac J Cancer Prev. 2006;7(2):309-12.

6. Zendehdel K, Sedigh Z, Hassanloo J, Nahvijou A. Audit of a Nationwide pathology-based cancer registry in Iran. Basic Clin Cancer Res. 2011;2:7-13.

7. Sadjadi A, Malekzadeh R, Derakhshan $\mathrm{MH}$, et al. Cancer occurrence in Ardabil: results of a population-based cancer registry from Iran. Int J Cancer. 2003;107(1):113-8.

8. Sadjadi A, Zahedi MJ, Darvish Moghaddam S, et al. The First PopulationBased Cancer Survey in Kerman Province of Iran. Iranian J Publ Health. 2007; 36(4):26-34.

9. Etemadi A, Sadjadi A, Semnani S, Nouraie SM, Khademi H, Bahadori M. Cancer registry in Iran: a brief overview. Arch Iran Med. 2008;11(5):577-80.

10. Hadji M, Nahvijou A, Seddighi Z, et al. Challenges to promoting populationbased cancer registration in Iran: a workshop report. Asian Pac J Cancer Prev. 2013;14(10):6189-93.

11. Kmet J, Mahboubi E. Esophageal cancer in the Caspian littoral of Iran: initial studies. Science. 1972;175(4024):846-53.

12. Mahboubi E, Kmet J, Cook PJ, Day NE, Ghadirian P, Salmasizadeh S. Oesophageal cancer studies in the Caspian Littoral of Iran: the Caspian cancer registry. Br J Cancer. 1973;28(3):197-214.

13. Kamangar F, Malekzadeh R, Dawsey SM, Saidi F. Esophageal cancer in Northeastern Iran: a review. Arch Iranian Medicine. Jan 2007;10(1):70-82.

14. Roshandel G, Semnani S, Sadjadi A, et al. Cancer Incidence in Islamic Republic of Iran, Golestan Province. In: Forman D, Bray F, Brewster DH, editors. Cancer Incidence in Five Continents, Vol. X (electronic version). Vol X. Lyon: International Agency for Research on Cancer; 2013.

15. Sadjadi A, Nouraie M, Mohagheghi MA, Mousavi-Jarrahi A, Malekezadeh R, Parkin DM. Cancer occurrence in Iran in 2002, an international perspective. Asian Pac J Cancer Prevention. 2005;6(3):359-63.

16. Somi MH, Farhang S, Mirinezhad SK, Naghashi S, Seif-Farshad M, Golzari M. Cancer in East Azerbaijan, Iran: results of a population-based cancer registry. Asian Pac J Cancer Prev. 2008;9(2):327-30.

17. Malekzadeh R, Derakhshan MH, Malekzadeh Z. Gastric cancer in Iran: epidemiology and risk factors. Arch Iranian Medicine. 2009;12(6):576-83.

18. Babaei M, Pourfarzi F, Yazdanbod A, et al. Gastric cancer in Ardabil, Iran--a review and update on cancer registry data. Asian Pac J Cancer Prev. 2010; 11(3):595-9. 
19. Zendehdel K, Marzban M, Nahvijou A, Jafari N. Six-fold Difference in the Stomach Cancer Mortality Rate between Northern and Southern Iran. Arch Iran Med. 2012;15(12):741-6.

20. Fritz A, Percy C, Jack A, et al. International classification of diseases for oncology (ICD-O) - 3rd edition, World Health Organization. 1st revision, 2013. http://apps.who.int/iris/bitstream/10665/96612/1/9789241548496_eng. pdf. Accessed 4 May 2016

21. Bray F, Znaor A, Cueva P, et al., eds. Planning and developing populationbased cancer registration in low- and middle-income settings. IARC Technical Publication No. 43, 2014. Published by the International Agency for Research on cancer. http://www.rho.org/files/IARC_Planning_ developing_cancer_registries_2014.pdf. Accessed 24 May 2016.

22. Boffetta P, Parkin DM. Cancer in developing countries. CA Cancer J Clin. 1994;44(2):81-90.

23. Black RJ, Bray F, Ferlay J, Parkin DM. Cancer incidence and mortality in the European Union: cancer registry data and estimates of national incidence for 1990. Eur J Cancer. 1997;33(7):1075-107.

24. Bray F, Guilloux A, Sankila R, Parkin DM. Practical implications of imposing a new world standard population. Cancer Causes Control. Mar 2002;13(2):175-82

25. Skeet RG. Cancer registration: principles and methods. Quality and quality control. IARC Sci Pub. 1991;95:101-7.

26. Demaret E, Ferlay J, Parkin M, Tycznski J, Whelan S. International Rules for Multiple Primary Cancers. Third ed. Lyone: France International Agency for Research on Cancer; 2004.

27. Bray F, Ferlay J, Laversanne M, et al. Cancer Incidence in Five Continents: Inclusion criteria, highlights from Volume $X$ and the global status of cancer registration. Int J Cancer. 2015;137(9):2060-71.

28. Roshandel G, Semnani S, Fazel A, et al. Building cancer registries in a lower resource setting: The 10-year experience of Golestan, Northern Iran. Cancer epidemiol. 2018;52:128-33.

29. Asthana S, Labani PS, Labani S. A review on cancer incidence in India from 25 population-based cancer registries. NTR Univ Health Sci. 2015:4:150-4.

30. Jedy-Agba E, Curado MP, Ogunbiyi O, et al. Cancer incidence in Nigeria: a report from population-based cancer registries. Cancer Epidemiol. Oct 2012; 36(5):e271-8.

31. Liu S, Chen Q, Quan P, et al. Cancer incidence and mortality in Henan province 2012. Chin J Cancer Res = Chung-kuo yen cheng yen chiu. 16; 28(3):275-85.

32. Al-Haddad BJ, Jedy-Agba E, Oga E, et al. Comparability, diagnostic validity and completeness of Nigerian cancer registries. Cancer Epidemiol. 2015; 39(3):456-64.

33. Somi MH, Naderi $\mathrm{N}$, Behshid M. Prevalence of gastrointestinal cancers in East Azerbaijan province. Pajoohandeh. 2004;39:165-9.

34. Somi MH, Golzari M, Farhang S, Naghashi S, Abdollahi L. Gastrointestinal cancer incidence in East Azerbaijan, Iran: update on 5 year incidence and trends. Asian Pac J Cancer Prev. 2014;15(9):3945-9.

35. Islami F, Kamangar F, Aghcheli K, et al. Epidemiologic features of upper gastrointestinal tract cancers in Northeastern Iran. Br J Cancer. 2004;90(7): 1402-6.

36. Mansour-Ghanaei F, Sokhanvar H, Joukar F, et al. Endoscopic findings in a mass screening program for gastric cancer in a high risk region - Guilan province of Iran. Asian Pac J Cancer Prev. 2012;13(4):1407-12.

37. Khodadost M, Yavari P, Khodadost B, et al. Estimating the Esophagus Cancer Incidence Rate in Ardabil, Iran: A Capture-Recapture Method. Iran J Cancer Prev. 2016;9(1):e3972.

38. Helicobacter, Cancer Collaborative G. Gastric cancer and Helicobacter pylori: a combined analysis of 12 case control studies nested within prospective cohorts. Gut. 2001:49(3):347-53.

39. Persson C, Jia Y, Pettersson H, Dillner J, Nyren $\mathrm{O}$, Ye WH. pylori seropositivity before age 40 and subsequent risk of stomach cancer: a glimpse of the true relationship? PloS one. 2011;6(3):e17404

40. Nyren O, Adami H-O. Stomach cancer. In: Adami H-O, Hunter D, Trichopoulos D, editors. Texbook of Cancer Epidemiology. New York: Oxford University Press; 2008.

41. Sadjadi A, Derakhshan MH, Yazdanbod A, et al. Neglected role of hookah and opium in gastric carcinogenesis: a cohort study on risk factors and attributable fractions. Int J Cancer. 2014;134(1):181-8.

42. Shakeri $\mathrm{R}$, Malekzadeh $\mathrm{R}$, Etemadi $\mathrm{A}$, et al. Opium: an emerging risk factor for gastric adenocarcinoma. Int J Cancer. 2013;133(2):455-61.
43. Islami F, Nasseri-Moghaddam S, Pourshams A, et al. Determinants of gastroesophageal reflux disease, including hookah smoking and opium use- a cross-sectional analysis of 50,000 individuals. PloS one. 2014;9(2): e89256.

44. Abnet CC, Saadatian-Elahi M, Pourshams A, et al. Reliability and validity of opiate use self-report in a population at high risk for esophageal cancer in Golestan, Iran. Cancer Epidemiol Biomarkers Prev. Jun 2004;13(6):1068-70.

45. Malekzadeh MM, Khademi H, Pourshams A, et al. Opium use and risk of mortality from digestive diseases: a prospective cohort study. Am J Gastroenterol. 2013;108(11):1757-65.

46. Zamani N, Hajifaraji M, Fazel-tabar Malekshah A, Keshtkar AA, Esmaillzadeh A, Malekzadeh R. A case-control study of the relationship between gastric cancer and meat consumption in Iran. Archlranian Med. Jun 2013;16(6):324-9.

47. Gandini S, Botteri $\mathrm{E}$, lodice $\mathrm{S}$, et al. Tobacco smoking and cancer: a metaanalysis. Int J Cancer. 2008;122(1):155-64.

48. Afshari M, Janbabaei G, Bahrami MA, Moosazadeh M. Opium and bladder cancer: A systematic review and meta-analysis of the odds ratios for opium use and the risk of bladder cancer. PloS one. 2017;12(6):e0178527.

49. Behmard S, Sadeghi A, Mohareri MR, Kadivar R. Positive association of opium addiction and cancer of the bladder. Results of urine cytology in 3,500 opium addicts. Acta Cytologica. 1981;25(2):142-6.

50. Khademi H, Malekzadeh R, Pourshams A, et al. Opium use and mortality in Golestan Cohort Study: prospective cohort study of 50,000 adults in Iran. Bmj. 2012;344:e2502.

51. Nemati S, Rafei A, Freedman ND, Fotouhi A, Asgary F, Zendehdel K Cigarette and Water-Pipe Use in Iran: Geographical Distribution and Time Trends among the Adult Population; A Pooled Analysis of National STEPS Surveys, 2006-2009. Arch Iranian Med. 2017;20(5):295-301.

52. Kamangar F, Shakeri R, Malekzadeh R, Islami F. Opium use: an emerging risk factor for cancer? Lancet Oncol. Feb 2014;15(2):e69-77.

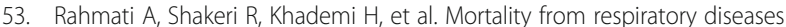
associated with opium use: a population-based cohort study. Thorax. 2017; 72(11):1028-34

54. Forman D, Bray F, Brewster DH, et al., eds. Cancer Incidence in Five Continents, Vol. X (electronic version). Vol X. Lyon: International Agency for Research on Cancer; 2013.

55. Bishehsari F, Mahdavinia M, Vacca M, Malekzadeh R, Mariani-Costantini R. Epidemiological transition of colorectal cancer in developing countries: environmental factors, molecular pathways, and opportunities for prevention. World J Gastroenterol. 2014;20(20):6055-72.

56. Malekzadeh R, Bishehsari F, Mahdavinia M, Ansari R. Epidemiology and molecular genetics of colorectal cancer in iran: a review. Arch Iranian Medicine. 2009;12(2):161-9.

57. Dolatkhah R, Somi MH, Bonyadi MJ, Asvadi Kermani I, Farassati F, Dastgiri S. Colorectal cancer in iran: molecular epidemiology and screening strategies. J Cancer Epidemiol. 2015;2015:643020.

58. Dolatkhah R, Somi MH, Kermani IA, et al. Increased colorectal cancer incidence in Iran: a systematic review and meta-analysis. BMC Public Health. 2015;15:997.

59. Policy statement and recommended actions for early detection of breast, cervical, colorectal, oral and prostate cancers in the Eastern Mediterranean Region, WHO-EM/NCD/138/E, 2016. http://applications.emro.who.int/docs/ Policy_statement_2016_en_19183.pdf. Accessed 4 May 2016.

60. Emadzadeh M, Shahidsales S, Mohammadian Bajgiran A, et al. Head and Neck Cancers in North-East Iran: A 25 year Survey. Iranian J Otorhinolaryngol. 2017;29(92):137-45.

61. Sadeghi H, Rafei M, Bahrami M, Haghdoost A, Shabani Y. Attributable risk fraction of four lifestyle risk factors of thyroid cancer: a meta-analysis. J Public Health. 2017:09:1-8.

62. Zhao ZG, Guo XG, Ba CX, et al. Overweight, obesity and thyroid cancer risk: a meta-analysis of cohort studies. J Int Med Research. 2012;40(6):2041-50.

63. Ma J, Huang M, Wang L, Ye W, Tong Y, Wang H. Obesity and risk of thyroid cancer: evidence from a meta-analysis of 21 observational studies. Med Science Monitor. 2015;21:283-91.

64. Semnani S, Sadjadi A, Fahimi S, et al. Declining incidence of esophageal cancer in the Turkmen Plain, eastern part of the Caspian Littoral of Iran: a retrospective cancer surveillance. Cancer Detection Prev. 2006;30(1):14-9.

65. Seifi S, Asvadi Kermani I, Dolatkhah R, et al. Prevalence of oral human papilloma virus in healthy individuals in East azerbaijan province of iran. Iran J Public Health. 2013;42(1):79-85. 
66. Khorasanizadeh F, Hassanloo J, Khaksar N, et al. Epidemiology of cervical cancer and human papilloma virus infection among Iranian women - analyses of national data and systematic review of the literature. Gynecologic Oncol. Feb 2013:128(2):277-81.

67. Mousavi S, Guya M, Ramazani R. Cancer Registration Report,for Year 2006. Tehran: Cancer Office, Center of Non-communicable Disease Control, Ministry of Health and Medical Education, I.R. of Iran;2007.

68. Haghshenas M, Golini-Moghaddam T, Rafiei A, Emadeian O, Shykhpour A, Ashrafi GH. Prevalence and type distribution of high-risk human papillomavirus in patients with cervical cancer: a population-based study. Infect Agent Cancer. 2013;8(1):20

69. Majidi A, Ghiasvand R, Hadji M, et al. Priority Setting for Improvement of Cervical Cancer Prevention in Iran. Int J Health Policy Manag. Nov 22 2015; 5(4):225-232.

Ready to submit your research? Choose BMC and benefit from:

- fast, convenient online submission

- thorough peer review by experienced researchers in your field

- rapid publication on acceptance

- support for research data, including large and complex data types

- gold Open Access which fosters wider collaboration and increased citations

- maximum visibility for your research: over $100 \mathrm{M}$ website views per year

At BMC, research is always in progress.

Learn more biomedcentral.com/submissions 